This article has been accepted for publication in Monthly Notices of the Royal Astronomical Society. (C: 2018 The Authors. Published by Oxford University Press on behalf of the Royal Astronomical Society. All rights reserved. 


\title{
ALMA view of a massive spheroid progenitor: a compact rotating core of molecular gas in an AGN host at $z=\mathbf{2 . 2 2 6}$
}

\author{
M. Talia, ${ }^{1,2 \star}$ F. Pozzi,,${ }^{1,2}$ L. Vallini, ${ }^{3}$ A. Cimatti, ${ }^{1,4}$ P. Cassata, ${ }^{5}$ F. Fraternali, ${ }^{1,6}$ \\ M. Brusa, ${ }^{1,2}$ E. Daddi, ${ }^{7}$ I. Delvecchio,${ }^{8}$ E. Ibar, ${ }^{5}$ E. Liuzzo,,${ }^{9}$ C. Vignali,,${ }^{1}$ M. Massardi,${ }^{9}$ \\ G. Zamorani, ${ }^{2}$ C. Gruppioni, ${ }^{2}$ A. Renzini, ${ }^{10}$ M. Mignoli, ${ }^{2}$ L. Pozzetti ${ }^{2}$ and \\ G. Rodighiero ${ }^{11}$ \\ ${ }^{1}$ Dipartimento di Fisica e Astronomia, Università di Bologna, Via Gobetti 93/2, I-40129, Bologna, Italy \\ ${ }^{2}$ INAF-Osservatorio Astronomico di Bologna, Via Gobetti 93/3, I-40129, Bologna, Italy \\ ${ }^{3}$ Nordita, KTH Royal Institute of Technology and Stockholm University, Roslagstullsbacken 23, SE-10691 Stockholm, Sweden \\ ${ }^{4}$ INAF-Osservatorio Astrofisico di Arcetri, Largo E. Fermi 5, I-50125, Firenze, Italy \\ ${ }^{5}$ Instituto de Fisica y Astronomía, Facultad de Ciencias, Universidad de Valparaíso, Gran Bretaña 1111, Playa Ancha, Valparaíso, Chile \\ ${ }^{6}$ University of Groningen, Kapteyn Astronomical Institute, Postbus 800, NL-9700 AV Groningen, the Netherlands \\ ${ }^{7}$ CEA, IRFU, DAp, AIM, Université Paris-Saclay, Université Paris Diderot, Sorbonne Paris Cité, CNRS, F-91191 Gif-sur-Yvette, France \\ ${ }^{8}$ Department of Physics, Faculty of Science, University of Zagreb, Bijenička cesta 32, 10000 Zagreb, Croatia \\ ${ }^{9}$ INAF-Istituto di Radioastronomia - Italian ARC, Via Piero Gobetti 101, I-40129 Bologna, Italy \\ ${ }^{10}$ INAF-Osservatorio Astronomico di Padova, Vicolo dell'Osservatorio 2, I-35122 Padova, Italy \\ ${ }^{11}$ Dipartimento di Fisica e Astronomia G. Galilei, Università di Padova, Vicolo dell'Osservatorio 3, I-35122 Padova PD, Italy
}

Accepted 2018 February 15. Received 2018 February 5; in original form 2017 November 16

\begin{abstract}
We present ALMA observations at 107.291 GHz (band 3) and 214.532 GHz (band 6) of GMASS 0953, a star-forming galaxy at $z=2.226$ hosting an obscured active galactic nucleus (AGN) that has been proposed as a progenitor of compact quiescent galaxies (QGs). We measure for the first time the size of the dust and molecular gas emission of GMASS 0953 that we find to be extremely compact $(\sim 1 \mathrm{kpc})$. This result, coupled with a very high interstellar medium (ISM) density $\left(n \sim 10^{5.5} \mathrm{~cm}^{-3}\right)$, a low gas mass fraction $(\sim 0.2)$, and a short gas depletion time-scale $(\sim 150 \mathrm{Myr})$, implies that GMASS 0953 is experiencing an episode of intense star formation in its central region that will rapidly exhaust its gas reservoirs, likely aided by AGN-induced feedback, confirming its fate as a compact QG. Kinematic analysis of the $\mathrm{CO}(6-5)$ line shows evidence of rapidly rotating gas $\left(V_{\mathrm{rot}}=320_{-53}^{+92} \mathrm{~km} \mathrm{~s}^{-1}\right)$, as observed also in a handful of similar sources at the same redshift. On-going quenching mechanisms could either destroy the rotation or leave it intact leading the galaxy to evolve into a rotating QG.
\end{abstract}

Key words: ISM: kinematics and dynamics - galaxies: active-galaxies: evolution-galaxies: high-redshift.

\section{INTRODUCTION}

Massive star-forming galaxies (SFGs) with centrally concentrated luminosity profiles at $z>2$ have been recently suggested by different authors to be the direct progenitors of compact quiescent galaxies (cQGs) at $z=1.5-3$ (e.g. Toft et al. 2007; Cassata et al. 2011; Wuyts et al. 2011; Whitaker et al. 2012; Barro et al. 2013; van der Wel et al. 2014; van Dokkum et al. 2015). Several theories have been proposed to achieve the high stellar densities observed in cQGs,

^E-mail: margherita.talia2@unibo.it including gas-rich mergers and/or disc instabilities (e.g. Tacconi et al. 2008; Zolotov et al. 2015; Tacchella et al. 2016), or in situ inside-out growth (e.g. Wellons et al. 2015; Lilly \& Carollo 2016).

Most scenarios predict the formation of a compact SFG (cSFG) as the last stage before quenching the star formation. Observationally, cSFGs candidates have been identified as being dense, compact, and dusty (Barro et al. 2013; Nelson et al. 2014; van Dokkum et al. 2015). The kinematics of the $\mathrm{H} \alpha$ emission line suggests that cSFGs have rotating disc of ionized gas slightly larger or comparable to the stellar distribution (van Dokkum et al. 2015; Wisnioski et al. 2018). From the comparison between the dynamical and stellar masses it is inferred that these galaxies must have low gas mass fractions and 
short gas depletion time-scales, as would be expected if they were soon to terminate their star formation.

A key element in the understanding of quenching mechanisms is a direct measurement of the size and mass content of the cold gas reservoirs that provide the fuel for star formation, but until now there have been only few of such measurements (e.g. Barro et al. 2016; Spilker et al. 2016; Popping et al. 2017; Tadaki et al. 2017b). cSFGs seem also to show a higher active galactic nucleus (AGN) incidence than the overall galaxy population at a fixed stellar mass (Kocevski et al. 2017; Wisnioski et al. 2018), suggesting that AGN activity might play a role in quenching, possibly through feedback provided by large-scale outflows (e.g. Genzel et al. 2014; Gilli et al. 2014; Brusa et al. 2015).

In order to investigate the gas properties of the progenitors of cQGs, in this letter we present ALMA spatially resolved observations of the dust continuum and $\mathrm{CO}$ lines emission of GMASS 0953, a heavily obscured AGN host selected from the GMASS sample (Kurk et al. 2013). We adopt a cosmology with $H_{0}=70 \mathrm{~km} \mathrm{~s}^{-1} \mathrm{Mpc}^{-1}, \Omega_{\mathrm{m}}=0.3, \Omega_{\Lambda}=0.7$, and assume a Chabrier (2003) initial mass function (IMF).

\section{GMASS 0953}

GMASS $0953^{1}$ (R.A. 03:32:31.48, Dec. $-27: 46: 23.40$ ) is a SFG at $z_{\mathrm{CO}}=2.2256$. It is detected in the 7Ms CDF-S X-ray maps (Luo et al. $2017)$ and hosts a heavily obscured $\left(N_{\mathrm{H}}>10^{24} \mathrm{~cm}^{-2}\right.$; Dalla Mura et al., in preparation) AGN with a rest-frame intrinsic luminosity (i.e. corrected for the obscuration) $L_{2-10 \mathrm{keV}} \sim 6.0 \times 10^{44} \mathrm{erg} \mathrm{s}^{-1}$. The target shows marginally extended emission in the $1.4 \mathrm{GHz}$ Very Large Array (VLA) radio continuum maps (Miller et al. 2013). The monochromatic $1.4 \mathrm{GHz}$ luminosity $L_{1.4 \mathrm{GHz}}=10^{24.84} \mathrm{~W} \mathrm{~Hz}^{-1}$ is consistent with radio emission predominantly arising from an AGN (Bonzini et al. 2012, 2013). Despite the clear presence of the AGN, the rest-frame ultraviolet (UV) spectrum remarkably does not show high-ionization emission lines (e.g. C IV $\lambda 1550$, Si IV $\lambda 1400$ ), likely because of the large obscuration of the nucleus (Cimatti et al. 2013). Optical lines ratios are consistent with a type II AGN, though shocks have also been proposed as an ionization mechanism (van Dokkum et al. 2005), supported by evidence of large-scale outflows in multiple gas phases (Cimatti et al. 2013; Förster Schreiber et al. 2014; Genzel et al. 2014, Loiacono et al., in preparation).

Following Delvecchio et al. (2014), we performed a multicomponent SED fitting to the available broad-band photometry with the SED3FIT code (Berta et al. 2013), that combines Bruzual \& Charlot (2003) stellar libraries, da Cunha, Charlot \& Elbaz (2008) IR-dust libraries, and Feltre, Hatziminaoglou \& Fritz (2012) torus + disc models. The full SED is shown in Fig. 1. We derive the stellar mass $M_{\star}=(1.15 \pm 0.1) \times 10^{11} \mathrm{M}_{\odot}$ and $\mathrm{SFR}_{\mathrm{IR}}=214 \pm 20 \mathrm{M}_{\odot} \mathrm{yr}^{-1}$, the latter assuming the Kennicutt (1998) relation [scaled to a Chabrier (2003); IMF)] between rest-frame $8-1000 \mu \mathrm{m} L_{\mathrm{IR}}$, corrected for the AGN contribution, and SFR. These values would place GMASS 0953 on the SFR-mass main sequence (MS; e.g. Rodighiero et al. 2011). A fit of the FIR points $(\lambda>24 \mu \mathrm{m})$ to a greybody gives values of $M_{\text {dust }}=(2.6 \pm 0.5) \times 10^{8} \mathrm{M}_{\odot}$ and $T_{\text {dust }}=38 \pm 2 \mathrm{~K}$, consistent with Popping et al. (2017).

Hubble Space Telescope (HST) images (Fig. 2; top: ACS/z band; bottom: WFC3/ $H$ band) show a compact morphology $\left(r_{\mathrm{e}, H \text { band }}=\right.$ $2.5 \mathrm{kpc}$; van der Wel et al. 2014) with a low-surface brightness tail

${ }^{1}$ a.k.a. K20-ID5, GS3-19791, 3D-HST GS30274 (e.g. Daddi et al. 2004; Förster Schreiber et al. 2009; Popping et al. 2017).

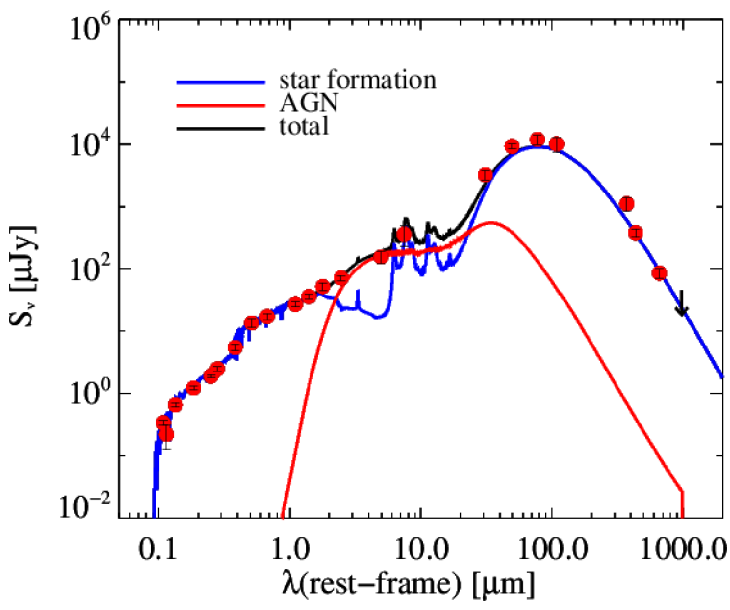

Figure 1. SED of GMASS 0953. The black line is the total best-fit model, while blue and red curves indicate, respectively, the star formation and AGN contributions. Red dots mark the observed photometry from: MUSIC (Grazian et al. 2006), SPITZER/MIPS (Magnelli et al. 2011), Herschel/PACS (Magnelli et al. 2013), and SPIRE (Roseboom et al. 2010), ALMA at $1.2 \mathrm{~mm}$ (Ueda et al. 2018), $1.4 \mathrm{~mm}$ (this work, Section 3), and $2.1 \mathrm{~mm}$ (Popping et al. 2017). The black arrow represents the $5 \sigma$ upper limit on the ALMA band 3 continuum obtained from the combined map of our data with those by Popping et al. (2017).
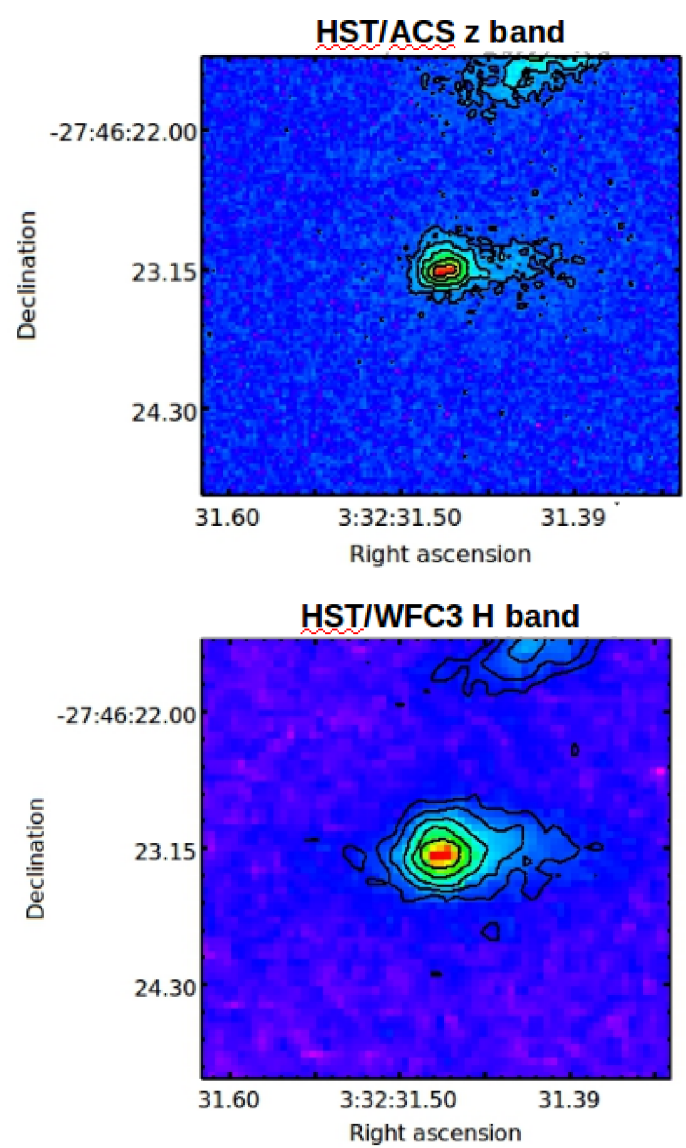

Figure 2. HST/ACS $z$ band (top; Giavalisco et al. 2004) and CANDELS HST/WFC3 $H$ band (bottom; Grogin et al. 2011; Koekemoer et al. 2011). The lower contours are at $3 \sigma$ level. The source to the north of our target is a foreground galaxy. 

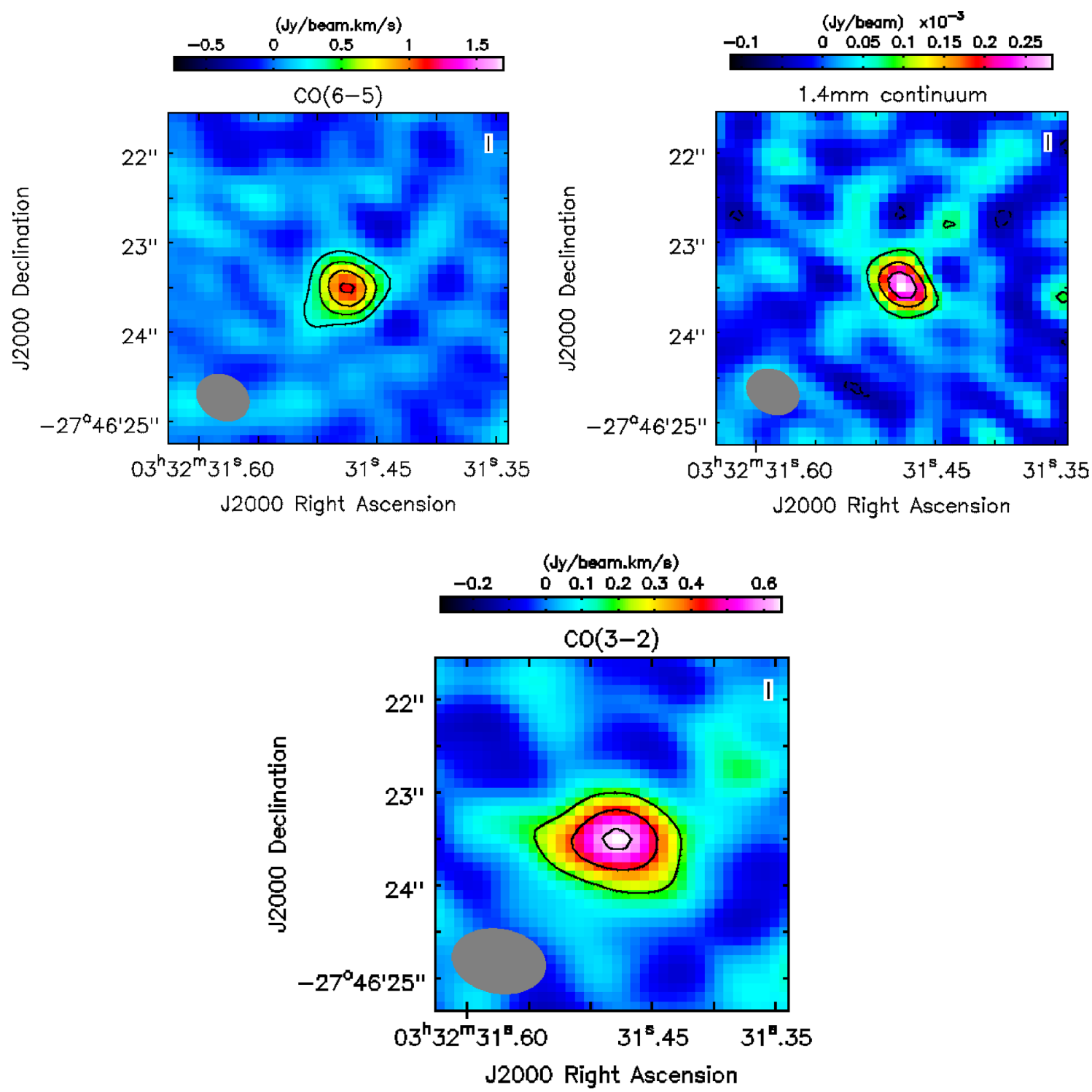

Figure 3. Moment 0 map of the $\mathrm{CO}(6-5)$ line (top, left), $1.4 \mathrm{~mm}$ continuum map (ALMA band 6; top, right), moment 0 map of the CO(3-2) line (bottom). The beam size is also shown in grey. In all images the lower continuous contours are at $3 \sigma$ level. The scales are the same as in Fig. 2, but no astrometric correction was applied (see Section 3).

to the west of the core that has been interpreted as either a merger remnant (van Dokkum et al. 2015) or a faint disc (Wisnioski et al. 2018).

\section{ALMA OBSERVATIONS}

ALMA observations were carried out in band 3 and 6, during Cycle 3 project 2015.1.01379.S (PI: P. Cassata) for a total integration time on source of $32 \mathrm{~min}$ and $1.3 \mathrm{~h}$, respectively, and an angular resolution of $0.6^{\prime \prime}$. The precipitable water vapour during the observations was between 1.4 and $3.1 \mathrm{~mm}$. We centred one spectral window of bandwidth $1.875 \mathrm{GHz}$ covering 3840 channels at 107.291 and $214.532 \mathrm{GHz}$, respectively, in band 3 and 6 to target $\mathrm{CO}(3-2)$ and $\mathrm{CO}(6-5)$ lines, and placed in each band, on line-free regions, other two spectral windows of bandwidth $1.875 \mathrm{GHz}$ covering 960 channels to target dust continuum.

The data were calibrated, imaged, and analysed using the standard ALMA pipeline and software package CASA (version 4.5.3;
McMullin et al. 2007). The calibrated data were cleaned interactively using masks at source position and setting a threshold of $3 \times$ rms noise level as measured on the dirty images. We adopted a Briggs weighting scheme (Briggs 1995) with a robust parameter of 0.2 and a channel width of $100 \mathrm{~km} \mathrm{~s}^{-1}$ as the best trade-off between sensitivity and spatial resolution, resulting in a clean beam of FWHM (full width at half-maximum) $=0.6^{\prime \prime} \times 0.5^{\prime \prime}$, with a position angle (PA) of $60^{\circ}$ in band 6 and of FWHM $=1.0^{\prime \prime} \times$ $0.7^{\prime \prime}$, with a PA of $80^{\circ}$ in band 3 .

We used the cleaned data cubes to produce continuum and line intensity maps (moment 0 ; Fig. 3) and to study the gas kinematics (Section 4). ALMA 1.4 mm continuum map (band 6) was obtained by averaging all the line-free channels in the data cube over a total velocity range of $\sim 4000 \mathrm{~km} \mathrm{~s}^{-1}$. The rms noise level is $\sigma_{1.4 \mathrm{~mm}}=$ $0.03 \mathrm{mJy} \mathrm{beam}^{-1}$. We do not have a significant continuum detection in band 3 . We also combined our band 3 continuum data with those from Popping et al. (2017) taken from the ALMA archive, after taking into account the different angular resolutions. We do not find 
a significant continuum detection also in the combined map. From the combined image we quote a $5 \sigma$ upper limit on band 3 flux of $\sim 0.05 \mathrm{mJy}$, that is consistent with the flux intensity predicted by the SED (Fig. 1).

Moment 0 maps of the $\mathrm{CO}(3-2)$ and $\mathrm{CO}(6-5)$ lines shown in Fig. 3 were obtained by integrating the line channels over the velocity range between -1000 and $1000 \mathrm{~km} \mathrm{~s}^{-1}$. In band 6, we first subtracted in the $u v$ plane the continuum with the task uvcontsub. The noise level measured in line-free channels is 0.15 and $0.10 \mathrm{mJy}$ beam $^{-1}$, respectively, in bands 3 and 6 .

We derived the source size and fluxes by fitting an elliptical Gaussian to the visibility data (task uvmodelfit), thus avoiding the uncertainty related to the cleaning parameters for these quantities. We measure line fluxes $I_{\mathrm{CO}(3-2)}=0.82 \pm 0.12 \mathrm{Jy} \mathrm{km}^{-1}$, consistent with that reported by Popping et al. (2017), and $I_{\mathrm{CO}(6-5)}=$ $1.21 \pm 0.13 \mathrm{Jy} \mathrm{km} \mathrm{s}^{-1}$ and $1.4 \mathrm{~mm}$ continuum flux $S_{1.4 \mathrm{~mm}}=$ $378 \pm 65 \mu \mathrm{Jy}$. Flux errors account for both measurement error and the 10 per cent absolute flux accuracy due to the calibrator.

While previous ALMA observations of GMASS 0953 (Popping et al. 2017) could not constrain the size of the molecular gas because of their lower angular resolution $\left(2^{\prime \prime}\right)$, we marginally resolve the target in band 6 . For the $\mathrm{CO}(6-5)$ line we measure a deconvolved FWHM $=0.18^{\prime \prime} \pm 0.06^{\prime \prime}$ (with an axis ratio of $1.0_{-0.3}^{+0.0}$ ), that corresponds to a radius $r_{\mathrm{CO}}=0.5 \times \mathrm{FWHM} \sim 0.75 \pm 0.25 \mathrm{kpc}$, and an intrinsic size of the continuum emission of FWHM $=0.30^{\prime \prime} \pm$ $0.09^{\prime \prime}\left(r_{1.4 \mathrm{~mm}}=1.24 \pm 0.37 \mathrm{kpc}\right)$, consistent with the $\mathrm{CO}(6-5)$ line within the errors.

We have performed Monte Carlo simulations in order to test the reliability of uvmodelfit errors on the size of our target. In particular, we simulated the observation of a mock galaxy with the same best-fit properties of our target, both in line and in continuum. We then created 100 realizations of the background noise to match our observations and measured the properties of the simulated sources using uvmodelfit. In both cases (i.e. line and continuum) the peak and the sigma of the distribution of the measurements are perfectly consistent with the uvmodel $\mathrm{f} i \mathrm{t}$ best-fit size and errors.

The emission centroids in ALMA and HST images are co-spatial, after accounting for the known systematic $\lesssim 0.5^{\prime \prime}$ shift in the NW direction between ALMA and HST positions in the CDFS (e.g. Rujopakarn et al. 2016; Dunlop et al. 2017; Ginolfi et al. 2017).

\subsection{ISM modelling}

GMASS 0953 had already been observed with ALMA by Popping et al. (2017) in bands 3 and 4, targeting $\mathrm{CO}(3-2), \mathrm{CO}(4-3)$, and [C I $](1-0)$ lines.

They derived a $\mathrm{CO}(1-0)$ luminosity of $L_{\mathrm{CO}}^{\prime}=2.1 \pm 0.2 \times 10^{10} \mathrm{~K}$ $\mathrm{km} \mathrm{s}^{-1} \mathrm{pc}^{2}$ assuming that the lines are all in the Rayleigh-Jeans limit and in local thermodynamic equilibrium (LTE). ${ }^{2}$ However, our new value of the $\mathrm{CO}(6-5)$ transition is different from what we would expect in the LTE approximation (Fig. 4), therefore we adopt an empirical method for estimating the $L_{\mathrm{CO}}^{\prime}$ luminosity. The shape of the observed CO-SLED of GMASS 0953 shows a strong similarity with the average SLEDs of supposedly similar sources, namely BzK (mostly MS galaxies at $z \sim 2$ ) and local ULIRGs (often hosting an AGN), normalized to the flux of the $J_{\text {up }}=3$ transition of our target (Fig. 4). Extrapolating the $\mathrm{CO}(1-0)$ transition from our observed $\mathrm{CO}(3-2)$ flux, assuming the average flux ratio from the appropriate literature

${ }^{2}$ The published value is actually a typo (Popping, private communication).

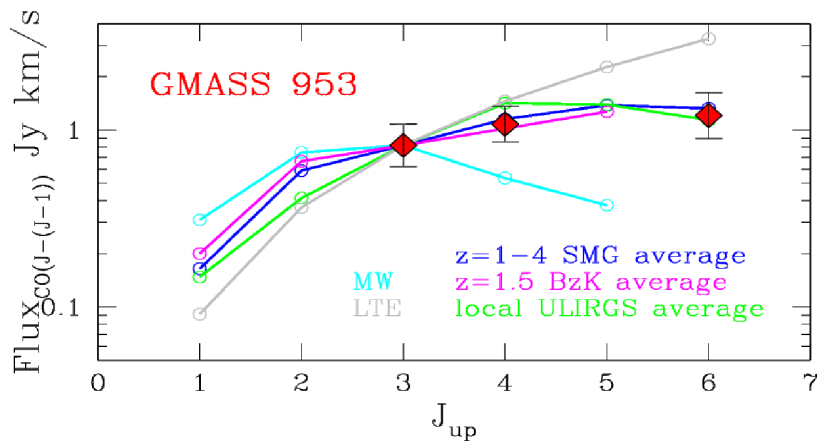

Figure 4. Observed CO-SLED of GMASS 0953 (red diamonds). We also plot, normalized to the $J_{\text {up }}=3$ transition of our source, the expected scaling in the LTE approximation, the Milky Way (Fixsen, Bennett \& Mather 1999), and the average values from different classes of objects, namely SMGs (Bothwell et al. 2013), BzK (Daddi et al. 2015), and ULIRGs (Papadopoulos et al. 2012). We added in quadrature a 10 per cent flux accuracy uncertainty to the $\mathrm{CO}(4-3)$ line flux of GMASS 0953, that was not accounted for in Popping et al. (2017) (Popping, private communication).

SLEDs, we estimate a flux $I_{\mathrm{CO}(1-0)}=0.17 \pm 0.03 \mathrm{Jy} \mathrm{km} \mathrm{s}{ }^{-1}$. A similar value would be derived normalizing the SLEDs to the $\mathrm{CO}(6-5)$ flux of our target.

From the CO(1-0) flux, following Solomon et al. (1997) we derive $L_{\mathrm{CO}}^{\prime}=(4.0 \pm 0.7) \times 10^{10} \mathrm{~K} \mathrm{~km} \mathrm{~s}^{-1} \mathrm{pc}^{2}$, about twice the value quoted by Popping et al. (2017). Assuming a CO-to- $\mathrm{H}_{2}$ conversion factor $\alpha_{\mathrm{CO}}=0.8 \mathrm{M}_{\odot} /\left(\mathrm{K} \mathrm{km} \mathrm{s}^{-1} \mathrm{pc}^{2}\right)$ we derive the gas mass: $M_{\mathrm{H}_{2}}=$ $(3.24 \pm 0.6) \times 10^{10} \mathrm{M}_{\odot}$, that is in good agreement with the estimate derived from the $\left[\mathrm{C}_{\mathrm{I}}\right]$ emission line and a factor of $\sim 4$ higher than that estimated from the dust mass (Popping et al. 2017). Our choice of $\alpha_{\mathrm{CO}}$ is motivated by the compactness of our source and its high SFR surface density (Section 5; see also Bolatto, Wolfire \& Leroy 2013).

Concerning the ISM physical properties, Popping et al. (2017) derived an estimate of the molecular gas density and the farUV (6-13.6 eV) radiation field flux from the comparison of the $\left[\mathrm{C}_{\mathrm{I}}\right] / \mathrm{CO}(4-3)$ intensity ratio of GMASS 0953 to the outputs of single photo-dissociation region (PDR) models from Kaufman et al. $(1999,2006)$. We did the same investigation with the code CLOUDY v17.00 (Ferland et al. 2017), adding our new observations. In particular, we run a grid of CLOUDY PDR models that span ranges in density and intensity of the UV radiation field that illuminates the cloud, assumed to be a 1D gas slab, and we linearly scaled with the SFR the CLOUdY default Cosmic ray ionization rate (see Bisbas, Papadopoulos \& Viti 2015; Vallini et al. 2018).

In Fig. 5, we show the predicted $\left[\mathrm{C}_{\mathrm{I}}\right] / \mathrm{CO}(6-5)$ luminosity ratio as a function of $G_{0}{ }^{3}$ and density $n$, highlighting the parameters space that give the observed $[\mathrm{C} \mathrm{I}] / \mathrm{CO}(6-5)$ (white) and $\mathrm{CO}(6-5) / \mathrm{CO}(4-3)$ (magenta) luminosity ratios.

It is evident that a single PDR with constant density and $G_{0}$ is not able to reproduce both luminosity ratios, because the two ratios do not trace the same parameters space. We argue that at least two components are needed to correctly model the observations, though a robust fit is not currently feasible because the degrees of freedom outnumber the data. Multiple phases are usually required to fit the ISM in local LIRGs and ULIRGs sources and in highredshift galaxies (e.g. Ward et al. 2003; Carilli et al. 2010; Danielson

\footnotetext{
${ }^{3} G_{0}$ is the flux in the far-ultraviolet band $(6-13.6 \mathrm{eV})$ scaled to that in the solar neighbourhood $\left(1.6 \times 10^{-3} \mathrm{erg} \mathrm{s}^{-1} \mathrm{~cm}^{-2}\right)$ (Habing 1968).
} 


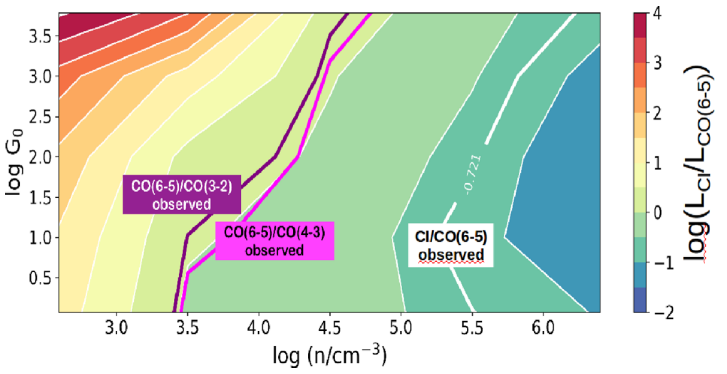

Figure 5. The $[\mathrm{C} \mathrm{I}] / \mathrm{CO}(6-5)$ luminosity ratio (expressed in $\mathrm{erg} \mathrm{s}^{-1} \mathrm{~cm}^{-2}$ ) as a function of the $G_{0}$ and density $n$. The observed values of [C I]/CO (6-5), $\mathrm{CO}(6-5) / \mathrm{CO}(4-3)$, and $\mathrm{CO}(6-5) / \mathrm{CO}(3-2)$ are marked, respectively, by the thick white, magenta, and purple contours.

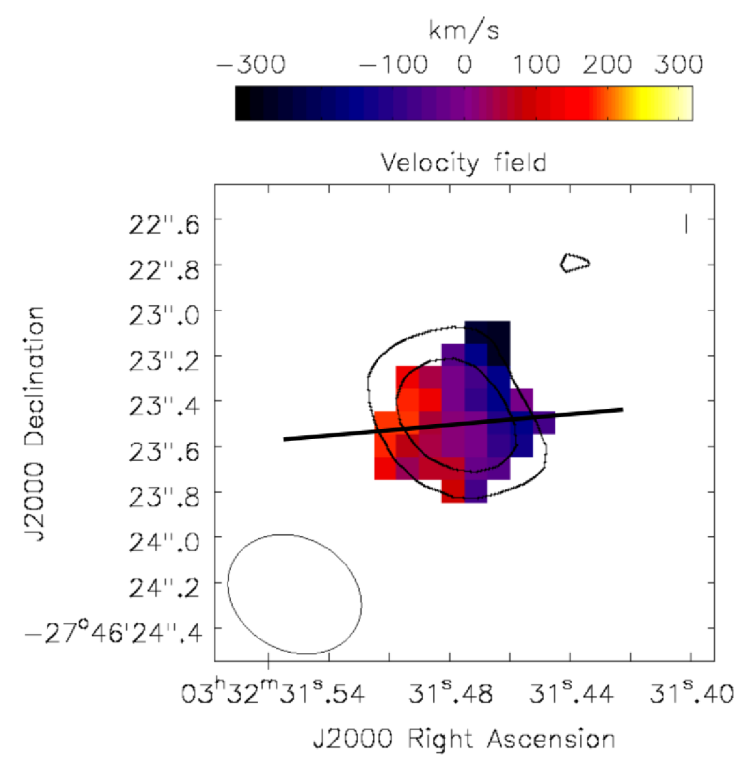

Figure 6. $\mathrm{CO}(6-5)$ velocity map with the continuum superimposed (black contours, $3 \sigma$ and $6 \sigma$, see Fig. 3). The black line shows the direction of the major axis.

et al. 2011; Daddi et al. 2015; Pozzi et al. 2017; Mingozzi et al. 2018), consisting in a diffuse, lower excitation component and a more concentrated, higher excitation gas. Our measurement of the $\mathrm{CO}(6-5)$ transition points towards the existence of a very dense ISM component with $n \sim 10^{5.5} \mathrm{~cm}^{-3}$.

We also point out that GMASS 0953 is hosting a Compton-thick AGN, and therefore higher excitation emission could also be associated with a dense $X$-ray dominated region (XDR), though higher-J $\mathrm{CO}$ lines would be needed to properly constrain its contribution (Meijerink, Spaans \& Israel 2007; van der Werf et al. 2010; Pozzi et al. 2017; Mingozzi et al. 2018).

\section{KINEMATICS}

The $\mathrm{CO}(3-2)$ and $\mathrm{CO}(6-5)$ lines have a FWHM of $733 \pm 98$ and $751 \pm 40 \mathrm{~km} \mathrm{~s}^{-1}$, respectively, that are consistent with the FWHM of $\mathrm{CO}(3-2), \mathrm{CO}(4-3)$, and [C I](1-0) reported by Popping et al. (2017).

We show the $\mathrm{CO}(6-5)$ velocity map in Fig. 6 and the positionvelocity (PV) diagram extracted along the major axis at a PA of 95 in Fig. 7. A velocity gradient is clearly detected. A merger system

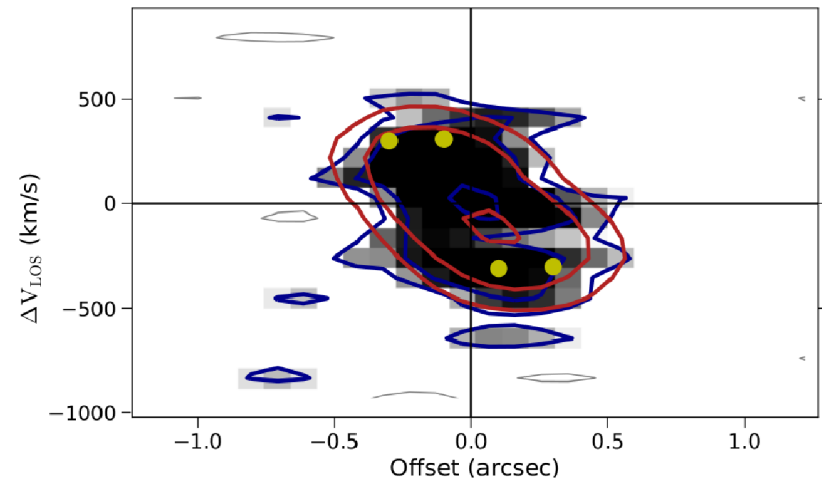

Figure 7. $\mathrm{CO}(6-5) \mathrm{PV}$ diagram extracted along the major axis at $\phi=95^{\circ}$, assuming an inclination of $75^{\circ}$. The iso-density contours $(2.5,5,10 \sigma)$ of the galaxy and BAROLO best-fit model are shown in blue and red, respectively. The yellow points mark the rotation curve.

in a coalescence phase observed at a favourable orientation could in principle originate such a gradient. However, this picture seems unlikely based on the absence of two distinct nuclei in the core of the high-resolution HST/ACS image (Fig. 2). Alternatively, the PV diagram could be the signature of a rotating disc of molecular gas. Possible evidence of a rotating disc of ionized gas in GMASS 0953 from the study of the $\mathrm{H} \alpha$ and [O III] $\lambda 5007$ emission lines kinematics has been also reported (Wisnioski et al. 2018; Loiacono et al., in preparation).

Under the assumption of a rotating disc we investigate the kinematic properties of the dense molecular gas traced by the $\mathrm{CO}(6-5)$ line with 3D-BAROLO (Di Teodoro \& Fraternali 2015), a tool for fitting 3D tilted-ring models to emission-line data cubes that takes into account the effect of beam smearing. We assumed a disc model with two rings and a ring width of $0.2^{\prime \prime}$, that is approximately half the clean beam size of the data cube. We fix the PA at $95^{\circ}$, that is the value that maximizes the spatial extension of the galaxy in the PV diagram. This value is consistent with both the photometric and kinematic $\mathrm{H} \alpha$ PAs as determined by HST imaging and KMOS data (van der Wel et al. 2014; Wisnioski et al. 2018). Then we run BAROLO leaving as free parameters the rotation velocity $\left(V_{\text {rot }}\right)$ and the intrinsic velocity dispersion $(\sigma)$ for different values of the inclination.

We derive a fiducial interval for the inclination (i.e. the range of values for which the model does not change significantly, as estimated from the residuals maps) between $60^{\circ}$ and $90^{\circ}$ and a best fit $V_{\text {rot }}=320_{-53}^{+92} \mathrm{~km} \mathrm{~s}^{-1}$, where the error includes both the formal error from the fit and the uncertainty from the variation of the inclination in our fiducial range. From our simulations, we also conclude that the model is quite insensitive to large variations in $\sigma$ due to the large channel width and estimate an upper limit of $\sigma$ $=140 \mathrm{~km} \mathrm{~s}^{-1}$. The best-fit model normalized to the azimuthally averaged flux in each ring is shown in red contours in Fig. 7. We also show the 1D spectrum extracted from the model, along with the $\mathrm{CO}(3-2)$ spectrum, in Fig. 8.

We note that the intrinsic $\mathrm{H} \alpha$ rotation curve presented in fig. 5 of Wisnioski et al. (2018) suggests an intrinsic velocity of $\sim 200 \mathrm{~km} \mathrm{~s}^{-1}$ on nuclear scale, broadly consistent with our results, and a lower velocity of $\sim 100 \mathrm{~km} \mathrm{~s}^{-1}$ at larger radii. Though it is difficult to make a direct comparison with the results by Wisnioski et al. (2018) because of the different approaches to deal with beam-smearing effects, we speculate that the combination of the two results might suggest a declining rotation curve of the inner regions of the gas 

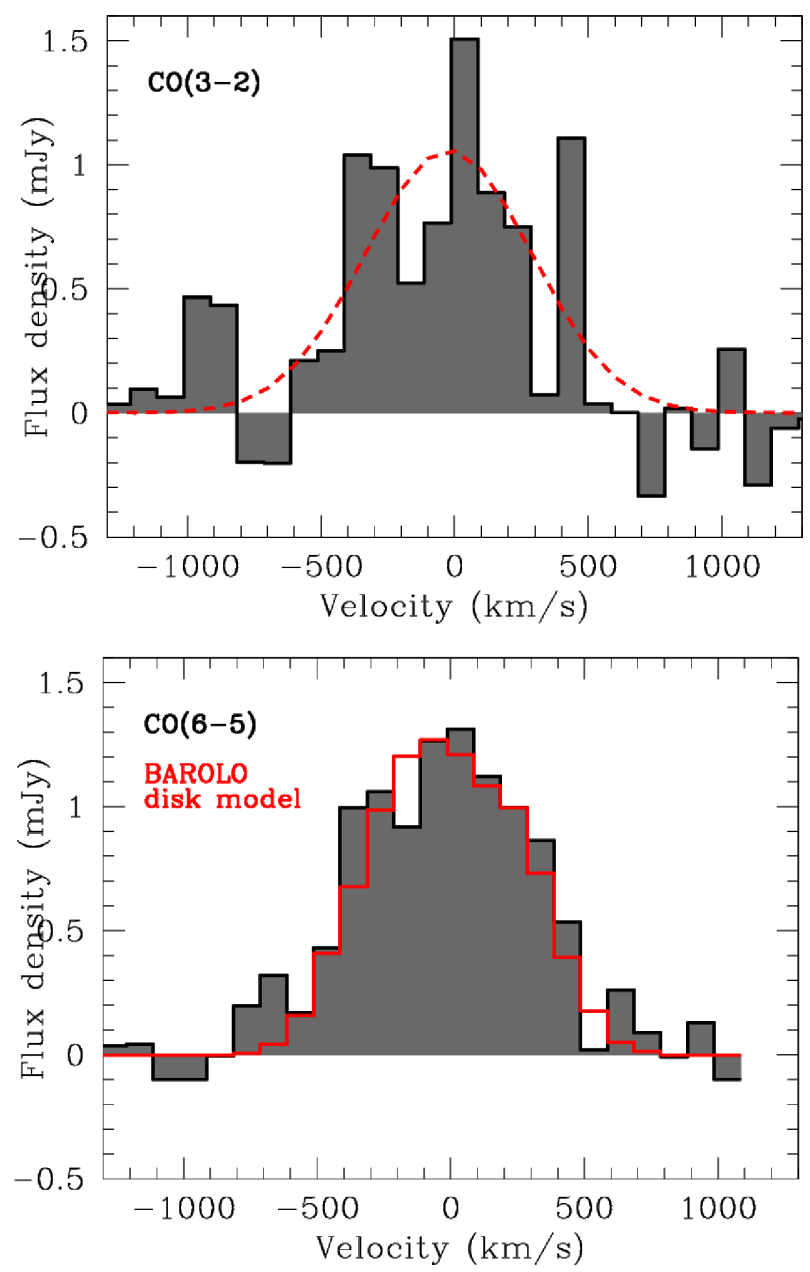

Figure 8. Velocity-integrated flux densities of the $\mathrm{CO}(3-2)$ (top) and $\mathrm{CO}(6-$ 5) (bottom). Both spectra were extracted from the respective data cubes in the region delimited by the $3 \sigma$ contour in the moment 0 map (see Fig. 3). The red dashed line in the top plot marks the Gaussian fit to the density profile. The red continuous line in the bottom plot is the disc model extracted from the 3D-BAROLo model-cube in same region as the source spectrum.

disc in GMASS 0953 as observed in massive early-type galaxies both locally and at high-redshift (Noordermeer et al. 2007; Genzel et al. 2017).

We note a $2.5 \sigma$ flux excess with respect to the disc model with an offset of $\Delta v \sim-700 \mathrm{~km} \mathrm{~s}^{-1}$ with respect to the line peak, also visible in the PV diagram. The velocity offset is consistent with the signatures of AGN-driven large-scale outflows in the neutral and ionized gas phases, namely the blueshift of rest-frame UV ISM absorption lines and the offset of a broad component detected in the [O III] $\lambda 5007$ emission line (Cimatti et al. 2013; Loiacono et al., in preparation). Popping et al. (2017) report that they do not find any signature of outflow in the flux density profile of the $\mathrm{CO}(4-3)$ line, that is detected at a similar significance level as the $\mathrm{CO}(6-5)$ line in this work. The lack of a flux excess in the lower-J observed transitions could indicate that the excitation ratio between the $\mathrm{CO}(6-$ 5 ) and lower-J transitions is higher in the possible outflow than in the rest of molecular gas in the host galaxy (e.g. Dasyra et al. 2016; Richings \& Faucher-Giguere 2018).

\section{DISCUSSION}

We have presented ALMA observations of GMASS 0953, an heavily obscured AGN host at $z \sim 2.226$. The $M_{\mathrm{H}_{2}}$ derived in Section 3.1 returns a gas fraction $M_{\mathrm{H}_{2}} /\left(M_{\mathrm{H}_{2}}+M_{\star}\right)=0.2$ and a gas depletion time-scale $\tau_{\text {depl }}=M_{\mathrm{H}_{2}} / \mathrm{SFR} \sim 150$ Myr. As pointed out by Popping et al. (2017), this value of $\tau_{\text {depl }}$ is much shorter than in more extended MS galaxies at the same redshift (Sargent et al. 2014; Scoville et al. 2017; Tacconi et al. 2018), but consistent with the values measured in off-MS galaxies, other cSFGs, and a few galaxies hosting an obscured AGN (e.g. Polletta et al. 2011; Brusa et al. 2015; Barro et al. 2016; Spilker et al. 2016; Tadaki et al. 2017b). We find evidence for a multiphase ISM in our galaxy and estimate the density of the higher excitation gas probed by the observed $\mathrm{CO}(6-5)$ line: $n \sim 10^{5.5} \mathrm{~cm}^{-3}$.

We measure a very compact radius $(\sim 1 \mathrm{kpc})$ for both the molecular gas and the dust emission, $\sim 2$ times smaller than the stellar distribution. We derive a gas mass surface density of $\Sigma_{M_{\mathrm{H}_{2}}}=$ $0.5 \times M_{\mathrm{H}_{2}} / \pi\left(r_{\mathrm{CO}}\right)^{2} \sim 9000 \mathrm{M}_{\odot} \mathrm{pc}^{-2}$. This value is similar to the typical stellar mass surface density of quiescent galaxies of similar stellar mass at the same redshift (Barro et al. 2017a). Considering that the SFR surface density at the radius of the dust continuum is $\Sigma_{\mathrm{SFR}}=0.5 \times \mathrm{SFR} / \pi\left(r_{1.4 \mathrm{~mm}}\right)^{2} \sim 22 \mathrm{M}_{\odot} \mathrm{yr}^{-1} \mathrm{kpc}^{-2}$ GMASS 0953 would lie at the high star formation and gas density end of the Kennicutt-Schmidt relation, consistent with local ULIRGs (e.g. Genzel et al. 2010).

From the aforementioned results we conclude that GMASS 0953, though formally lying on the MS of SFGs, has a lower gas content than MS galaxies with the same stellar mass and is consuming it much more rapidly in a very compact core (Elbaz et al. 2017). On short time-scales this galaxy will likely exhaust its gas reservoirs and become a cQG. This scenario, consistent with previous analysis of ISM properties and optical emission lines kinematics (van Dokkum et al. 2015; Popping et al. 2017; Wisnioski et al. 2018), finds a further confirmation from our direct measurement of the extremely compact size of the star-forming region and the molecular gas of GMASS 0953 (Gilli et al. 2014; Barro et al. 2016; Tadaki et al. 2017a,b; Brusa et al. 2018). With our data we are unable to discriminate between the different mechanisms that could have originated such a compact core, i.e. a merger occurred in the past (e.g. Tacconi et al. 2008; Wellons et al. 2015), disc instabilities (e.g. Dekel $\&$ Burkert 2014; Ceverino et al. 2015; Zolotov et al. 2015), or in situ secular processes (e.g. Wellons et al. 2015; van Dokkum et al. 2015), though recent studies tend to favour dissipative formation mechanisms to explain the smaller size of the nuclear region of intense star formation with respect to the stellar distribution (Barro et al. 2016; Tadaki et al. 2017a).

AGN activity is also advocated as a quenching mechanism for cSFGs in addition to the gas consumption provided by the strong star formation activity (Barro et al. 2013), both likely triggered by the same mechanism that led to the formation of the compact core (Kocevski et al. 2017). In particular, because of its compactness and the presence of a luminous, obscured AGN, GMASS 0953 is consistent with the 'quasar mode' postulated by Hopkins et al. (2006) where the AGN quenches the star formation within the host galaxy through feedback mechanisms (see also Rangel et al. 2014, and Lapi et al. 2018), e.g. fast large-scale outflows as those that have been observed in different gas phases of GMASS 0953 (Cimatti et al. 2013; Förster Schreiber et al. 2014; Genzel et al. 2014, Loiacono et al., in preparation), tentatively including the molecular one. 
GMASS 0953 is also one of the first cases in which, thanks to the quality of the data, we are able to measure the rapid rotation ( $V_{\text {rot }}=$ $320_{-53}^{+92} \mathrm{~km} \mathrm{~s}^{-1}$ ) of the molecular gas disc in the core (Tadaki et al. 2017b; Barro et al. 2017b; Brusa et al. 2018), predicted by some simulations before the gas is completely depleted (Shi et al. 2017), though it is not yet clear if this is a common feature in all cQGs progenitors (e.g. Spilker et al. 2016). The observation of stellar rotation in cQGs (Newman, Belli \& Ellis 2015; Toft et al. 2017) could indicate that cSFGs cores might retain their rotation after the quenching processes.

In conclusion, in this work we have highlighted the importance of spatially resolved ALMA observations for the study of a prototypical progenitor of cQGs, likely caught in the act of quenching through the combined action of efficient compact nuclear star formation activity and AGN feedback.

\section{ACKNOWLEDGEMENTS}

This paper makes use of the following ALMA data: ADS/JAO. ALMA\#2015.1.01379.S (PI: Cassata); \#2015.1.00228.S (PI: Popping). ALMA is a partnership of ESO (representing its member states), NSF (USA), and NINS (Japan), together with NRC (Canada), NSC and ASIAA (Taiwan), and KASI (Republic of Korea), in cooperation with the Republic of Chile. The Joint ALMA Observatory is operated by ESO, AUI/NRAO, and NAOJ. We acknowledge extensive support in data reduction and analysis from the ALMA Regional Centre in Bologna. MT gratefully thanks E. Di Teodoro for his support with the 3D-BARolo code, P. Popesso for her warm hospitality in Munich during the writing of this paper, L. Pantoni for providing the dust mass and temperature of GMASS 0953, G. Popping, R. Decarli, R. Paladino, and A. Lapi for useful discussions. PC acknowledges support from CONICYT through the project FONDECYT regular 1150216. MB acknowledges support from the FP7 Career Integration Grant 'eEASy' (CIG 321913). FP, CG, AR, LP, and GR acknowledge funding from the INAF PRIN-SKA 2017 programme 1.05.01.88.04. EI acknowledges partial support from FONDECYT through grant $N^{\circ} 1171710$. The authors thank the anonymous referee for constructive comments that helped to improve the presentation of the results.

\section{REFERENCES}

Barro G. et al., 2013, ApJ, 765, 104

Barro G. et al., 2016, ApJ, 827, L32

Barro G. et al., 2017a, ApJ, 840, 47

Barro G. et al., 2017b, ApJ, 851, L40

Berta S. et al., 2013, A\&A, 551, A100

Bisbas T. G., Papadopoulos P. P., Viti S., 2015, ApJ, 803, 37

Bolatto A. D., Wolfire M., Leroy A. K., 2013, ARA\&A, 51, 207

Bonzini M. et al., 2012, ApJS, 203, 15

Bonzini M., Padovani P., Mainieri V., Kellermann K. I., Miller N., Rosati

P., Tozzi P., Vattakunnel S., 2013, MNRAS, 436, 3759

Bothwell M. S. et al., 2013, MNRAS, 429, 3047

Briggs D. S., 1995, AAS Meeting Abstracts, p. 1444

Brusa M. et al., 2015, A\&A, 578, A11

Brusa M. et al., 2018, A\&A, preprint (arXiv:1712.04505)

Bruzual G., Charlot S., 2003, MNRAS, 344, 1000

Carilli C. L. et al., 2010, ApJ, 714, 1407

Cassata P. et al., 2011, ApJ, 743, 96

Ceverino D., Dekel A., Tweed D., Primack J., 2015, MNRAS, 447, 3291

Chabrier G., 2003, PASP, 115, 763

Cimatti A. et al., 2013, ApJ, 779, L13

da Cunha E., Charlot S., Elbaz D., 2008, MNRAS, 388, 1595

Daddi E. et al., 2004, ApJ, 600, L127
Daddi E. et al., 2015, A\&A, 577, A46

Danielson A. L. R. et al., 2011, MNRAS, 410, 1687

Dasyra K. M., Combes F., Oosterloo T., Oonk J. B. R., Morganti R., Salomé P., Vlahakis N., 2016, A\&A, 595, L7

Dekel A., Burkert A., 2014, MNRAS, 438, 1870

Delvecchio I. et al., 2014, MNRAS, 439, 2736

Di Teodoro E. M., Fraternali F., 2015, MNRAS, 451, 3021

Dunlop J. S. et al., 2017, MNRAS, 466, 861

Elbaz D. et al., 2017, A\&A, preprint (arXiv:1711.10047)

Feltre A., Hatziminaoglou E., Fritz J., 2012, MNRAS, 426, 120

Ferland G. J. et al., 2017, Rev. Mex. Astron. Astrofis., 53, 385

Fixsen D. J., Bennett C. L., Mather J. C., 1999, ApJ, 526, 207

Förster Schreiber N. M. et al., 2009, ApJ, 706, 1364

Förster Schreiber N. M. et al., 2014, ApJ, 787, 38

Genzel R. et al., 2010, MNRAS, 407, 2091

Genzel R. et al., 2014, ApJ, 796, 7

Genzel R. et al., 2017, Nature, 543, 397

Giavalisco M. et al., 2004, ApJ, 600, L93

Gilli R. et al., 2014, A\&A, 562, A67

Ginolfi M. et al., 2017, MNRAS, 468, 3468

Grazian A. et al., 2006, A\&A, 449, 951

Grogin N. A. et al., 2011, ApJS, 197, 35

Habing H. J., 1968, Bull. Astron. Inst. Neth., 19, 421

Hopkins P. F., Hernquist L., Cox T. J., Di Matteo T., Robertson B., Springel V., 2006, ApJS, 163, 1

Kaufman M. J., Wolfire M. G., Hollenbach D. J., Luhman M. L., 1999, ApJ, 527,795

Kaufman M. J., Wolfire M. G., Hollenbach D. J., 2006, ApJ, 644, 283

Kennicutt R. C., Jr, 1998, ARA\&A, 36, 189

Kocevski D. D. et al., 2017, ApJ, 846, 112

Koekemoer A. M. et al., 2011, ApJS, 197, 36

Kurk J. et al., 2013, A\&A, 549, A63

Lapi A. et al., 2018, ApJ, preprint (arXiv:1803.04734)

Lilly S. J., Carollo C. M., 2016, ApJ, 833, 1

Luo B. et al., 2017, ApJS, 228, 2

Magnelli B., Elbaz D., Chary R. R., Dickinson M., Le Borgne D., Frayer D. T., Willmer C. N. A., 2011, A\&A, 528, A35

Magnelli B. et al., 2013, A\&A, 553, A132

McMullin J. P., Waters B., Schiebel D., Young W., Golap K., 2007, in Shaw R. A., Hill F., Bell D. J., eds, Astronomical Data Analysis Software and Systems XVI, Vol. 376. Astronomical Society of the Pacific, San Francisco, CA, p. 127

Meijerink R., Spaans M., Israel F. P., 2007, A\&A, 461, 793

Miller N. A. et al., 2013, ApJS, 205, 13

Mingozzi M. et al., 2018, MNRAS, 474, 3640

Nelson E. et al., 2014, Nature, 513, 394

Newman A. B., Belli S., Ellis R. S., 2015, ApJ, 813, L7

Noordermeer E., van der Hulst J. M., Sancisi R., Swaters R. S., van Albada T. S., 2007, MNRAS, 376, 1513

Papadopoulos P. P., van der Werf P., Xilouris E., Isaak K. G., Gao Y., 2012, ApJ, 751, 10

Polletta M., Nesvadba N. P. H., Neri R., Omont A., Berta S., Bergeron J., 2011, A\&A, 533, A20

Popping G. et al., 2017, A\&A, 602, A11

Pozzi F., Vallini L., Vignali C., Talia M., Gruppioni C., Mingozzi M., Massardi M., Andreani P., 2017, MNRAS, 470, L64

Rangel C. et al., 2014, MNRAS, 440, 3630

Richings A. J., Faucher-Giguere C.-A., 2018, MNRAS, 474, 3673

Rodighiero G. et al., 2011, ApJ, 739, L40

Roseboom I. G. et al., 2010, MNRAS, 409, 48

Rujopakarn W. et al., 2016, ApJ, 833, 12

Sargent M. T. et al., 2014, ApJ, 793, 19

Scoville N. et al., 2017, ApJ, 837, 150

Shi J., Lapi A., Mancuso C., Wang H., Danese L., 2017, ApJ, 843, 105

Solomon P. M., Downes D., Radford S. J. E., Barrett J. W., 1997, ApJ, 478, 144

Spilker J. S., Bezanson R., Marrone D. P., Weiner B. J., Whitaker K. E., Williams C. C., 2016, ApJ, 832, 19 
Tacchella S., Dekel A., Carollo C. M., Ceverino D., DeGraf C., Lapiner S., Mandelker N., Primack J. R., 2016, MNRAS, 458, 242

Tacconi L. J. et al., 2008, ApJ, 680, 246

Tacconi L. J. et al., 2018, ApJ, 853, 179

Tadaki K.-i. et al., 2017a, ApJ, 834, 135

Tadaki K.-i. et al., 2017b, ApJ, 841, L25

Toft S. et al., 2007, ApJ, 671, 285

Toft S. et al., 2017, Nature, 546, 510

Ueda Y. et al., 2018, ApJ, 853, 24

Vallini L., Pallottini A., Ferrara A., Gallerani S., Sobacchi E., Behrens C., 2018, MNRAS, 473, 271

van der Wel A. et al., 2014, ApJ, 788, 28

van der Werf P. P. et al., 2010, A\&A, 518, L42 van Dokkum P. G., Kriek M., Rodgers B., Franx M., Puxley P., 2005, ApJ, 622, L13

van Dokkum P. G. et al., 2015, ApJ, 813, 23

Ward J. S., Zmuidzinas J., Harris A. I., Isaak K. G., 2003, ApJ, 587, 171

Wellons S. et al., 2015, MNRAS, 449, 361

Whitaker K. E., Kriek M., van Dokkum P. G., Bezanson R., Brammer G., Franx M., Labbe Ivo, 2012, ApJ, 745, 179

Wisnioski E. et al., 2018, ApJ, 855, 97

Wuyts S. et al., 2011, ApJ, 742, 96

Zolotov A. et al., 2015, MNRAS, 450, 2327

This paper has been typeset from a $\mathrm{T}_{\mathrm{E}} \mathrm{X} / \mathrm{L} \mathrm{T} \mathrm{E} \mathrm{X}$ file prepared by the author. 